\title{
Propagation characteristics and wavelength tuning of amplified spontaneous emission from dye-doped polymer
}

\author{
K. Geetha, M. Rajesh, V. P. N. Nampoori, C. P. G. Vallabhan, and P. Radhakrishnan
}

\begin{abstract}
The propagation characteristics of amplified spontaneous emission (ASE) through a rhodamine 6 G-doped polymethyl methacrylate freestanding film waveguide were studied. This was done by shifting it excitation stripe horizontally along a transversely pumped waveguide. By this method, we could tme bs ASE wavelength. The maximum tunability thus obtained was $-18 \mathrm{~nm}$ with a pump stripe length $\alpha$ $6 \mathrm{~mm}$. 2006 Optical Society of America
\end{abstract}

OCIS codes: $230.7370,300.2140,140.3580,250.5460$.

\section{Introduction}

Organic dye lasers have become a subject of intensive research ever since Sorokin and Lankard reported the stimulated emission from an organic dye solution. ${ }^{1}$ The use of solid matrix for dye lasers eliminates many of the common problems associated with liquid systems such as concentration variation due to the evaporation of solvents. The first attempts to develop solid-state dye lasers were reported in the late 1960 s. $^{2,3}$ Most of the recent work has been done using either polymers ${ }^{4,5}$ or silica gels $5^{6.7}$ as the host media. Polymeric materials in particular offer advantages such as ease of processing, which permits fabrication of devices of virtually any shape and potentially very low cost. Organic polymers such as poly(methylmethacrylate) 8.9 (PMMA) or poly(acrylic acid) ${ }^{10}$ (PA) have been widely used as host material for laser dyes.

Dye-doped solid-state waveguide lasers provide low-cost, high-efficiency tunable coherent light sources. Waveguide structures provides long gain length and optical confinement, which offer reduction of the lasing threshold. Conventionally, wavelength

K. Geetha, V. P. N. Nampoori, and P. Radhakrishnan are with the International School of Photonies, Cochin University of Science and Technology, Cochin-22, India. M. Rajesh is with the International School of Photonics, Cochin University of Science and Technology, Cochin-22, India, and the Centre of Excellence in Laser and Optoelectronic Science (CELOS), Cochin University of Science and Technology, Cochin-22, India. C. P. G. Vallabhan is with the Centre of Excellence in Laser and Optuelectronic Science (CELOS), Cochin University of Science and Technology, Cochin-22, India.

Received 6 June 2005; revised 12 September 2005; accepted 14 September 2005.

0003-6935/06/040764-06\$15.00/0

(1) 2006 Optical Society of America tuning is achieved by the use of distributed Bn reflectors as coupling mirrors in resonant cavitie li In external cavity solid-state lasers, wide-rang: wavelength tuning has been achieved by the use of grating as the dispersion element. A tunability d approximately $53 \mathrm{~nm}$ was obtained with a multipit prism-grating solid-state dye laser where the gie medium was a rhodamine 6G (Rh6G)-doped motified PMMA with trapezoidal geometry. ${ }^{12}$ Multimode ing and wideband tuning in sol-gel distributed back waveguide lasers have also been reported. 1 . In these lasers, tuning was achieved either by ing the period of gain modulation ${ }^{13,14}$ or by varyis the temperature of the gain medium, thereby varjug its refractive index. ${ }^{15}$ By using the first metbod, tunability of more than $30 \mathrm{~nm}$ was achieved. By ing the latter method, with a Rh6G-doped sol-ged silica waveguide, $17 \mathrm{~nm}$ tunability was obtained is varying the temperature from $21^{\circ} \mathrm{C}$ to $58^{\circ} \mathrm{C}$ wh with Rh6G-doped PMMA waveguide, $6 \mathrm{~nm}$ tunating was obtained by varying temperature from $22 \%$ to $98^{\circ} \mathrm{C}$

For most dyes, the absorption and emission bans overlap and the short wavelength fluorescence is sorbed. For such dyes the observed fluorescence peatis shifted to longer wavelengths, at higher concentr tions. Thus wavelength tuning can also be achieved th changing the dye concentration. ${ }^{16}$

In high-gain structures, under strong pumpin conditions, consideration of the phenomenon of at plified spontaneous emission (ASE) becomes very portant for understanding the behavior of sxis systems. ASE is a phenomenon where the spontan ously emitted light gets amplified as it propagen along the gain medium, and even without feedbed the emitted radiation exhibits laserlike propertie 
th as low divergence and spectral narrowing. A hnique for tuning the ASE wavelength by controlg the waveguide thickness has been reported for gugated polymer films ${ }^{17}$ as well as for dye-doped lid-state waveguides. ${ }^{18} \mathrm{~A}$ wide-range tunability of and $30 \mathrm{~nm}$ was observed in these two cases. Organic dyes exhibit a concentration-dependent shift of the peak emission wavelength. ${ }^{16}$ Similar hhift was observed for the spectra taken from usversely pumped dye-doped fibers and wavesites when the excitation spot position was shifted me length of the fiber or waveguide by transing either the source or the waveguide. 19-21 Using inilar technique, we investigated the wavelength ung in a transversely pumped Rh6G-doped PMMA ta waveguide by observing the wavelength changes IASE. The cross section of the pump beam was in vorm of a stripe. The position of excitation stripe shifted in such a way that the ASE from the end the pump stripe is guided along different lengths of a waveguide. A tunability of $\sim 18 \mathrm{~nm}$ was obtained then the distance between the waveguide edge and it pump stripe edge was varied from 0 to $15 \mathrm{~mm}$. th main advantage of the present technique is that itulk optics are used for tuning. By simultaneous atral measurement from both ends of the pumped meguide, simultaneous tuning of dual wavelength vission can also be achieved, details of which will be wished elsewhere.

\section{Experiment}

Ineriments were conducted on freestanding thin Is of PMMA doped with Rh6G. PMMA, which is tmost frequently used polymer host for dye lasers, the best optical transparency in the visible range. RhG6, the best known of all laser , has been frequently investigated in solid-state lasers in a variety of solid hosts, on account of its fluorescence quantum yield, low intersystem using rate, and low excited-state absorption at pump and lasing wavelengths. The samples at prepared by dissolving PMMA and Rh6G in whylethylketone, with $0.5 \mathrm{mM}$ dye concentration. ms of $50 \mu \mathrm{m}$ thickness were tape cast on glass from this solution. When the solvent is fully oporated, freestanding films could be peeled off the sheet. The films were then cut into the size I. $\times 2 \mathrm{~cm}$.

The samples were transversely pumped using los pulses from a frequency doubled Nd:YAG laser in $\mathrm{nm}, 10 \mathrm{~Hz}$ ). A set of calibrated neutral density were used for varying the pump energy. The menergy was varied from 0.03 to $9.1 \mathrm{~mJ} /$ pulse. weam was focused into a narrow stripe of approxstely $50 \mu \mathrm{m}$ width. The stripe length was varied eng an adjustable slit.

When ASE occurs in a long narrow stripe, most of the is is emitted at the ends of the stripe. The emission in the sample was collected by a fiber and directed to $10.5 \mathrm{~m}$ spectrometer with a cooled CCD array. The between the collecting fiber and the reguide edge was $1 \mathrm{~cm}$. A slit was kept in front of

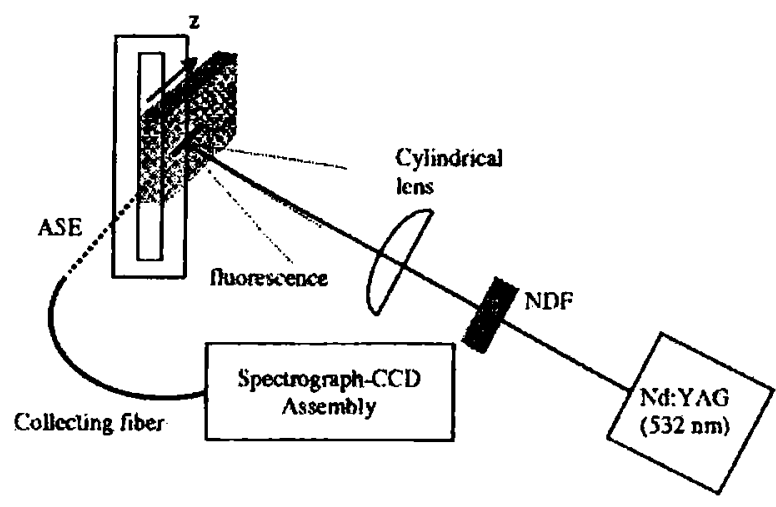

Fig. 1. Excitation and light collecting schemes.

the fiber so that the emission from the front and back surfaces was blocked. The sample was kept on a translator so as to enable horizontal shifting of the excitation stripe along the length of the waveguide. Initially the excitation stripe was formed at the edge $(z=0)$ of the waveguide and the emission spectra for various pump energies were recorded. Then the stripe position was shifted, thereby varying $z$-the distance between one end of the pump stripe and the observation edge of the film (Fig. 1). Observations were made for stripe lengths of 2,4 , and $6 \mathrm{~mm}$.

\section{Results and Discussion}

\section{A. Observation of Amplified Spontaneous Emission}

First we recorded the emission spectra for $z=0$, at various pump intensities with a constant pump stripe of length $2 \mathrm{~mm}$ (Fig. 2).

At low pump energies, the emission spectrum is broad. But above the pump energy of $0.31 \mathrm{~mJ} /$ pulse, the peak of the spectrum at approximately $565 \mathrm{~nm}$ grew strongly. At $E=9.1 \mathrm{~mJ} /$ pulse, a narrow spec-

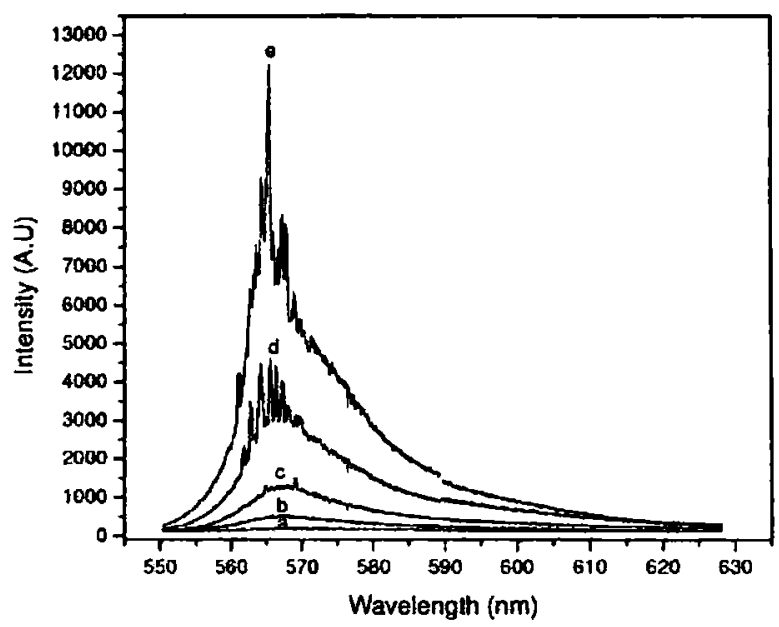

Fig. 2. Emission from the waveguide edge for stripe length $2 \mathrm{~mm}$, for various pump energies: (a) $0.031 \mathrm{~mJ} /$ pulse; (b) $0.10 \mathrm{~mJ} /$ pulse; (c) $0.31 \mathrm{~mJ} /$ pulse; (d) $1 \mathrm{~mJ} /$ pulse; (e) $9.1 \mathrm{~mJ} /$ pulse. 


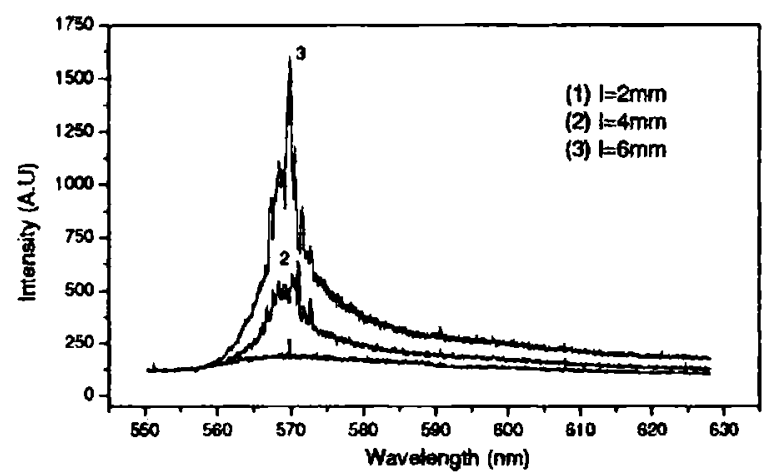

(a)
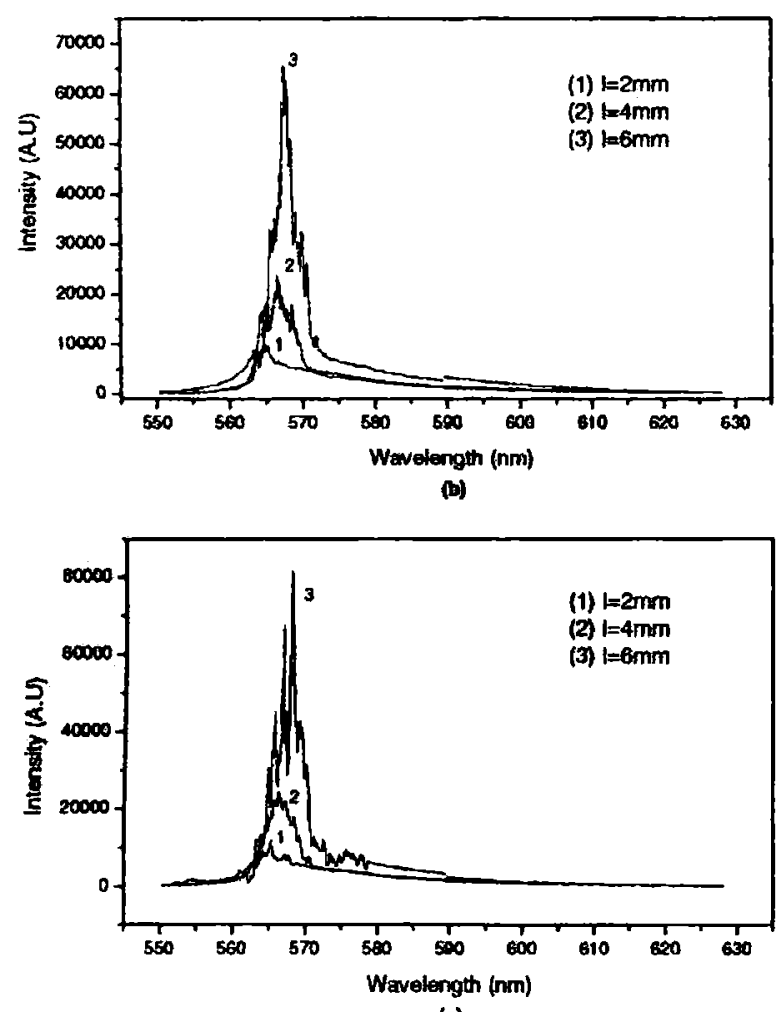

(c)

Fig. 3. Spectral narrowing with increase in stripe length for various pump energies: (a) $E=0.031 \mathrm{~mJ} /$ pulse; (b) $E=3.1 \mathrm{~mJ} /$ pulse; (c) $E=9.1 \mathrm{~mJ} /$ pulse.

trum centered at $565.4 \mathrm{~nm}$ with a FWHM of only $\sim 5 \mathrm{~nm}$ was obtained.

Spectral narrowing of the emitted radiation can be due to several reasons. To confirm the phenomenon responsible for spectral narrowing, we varied the length of the excitation stripe. If ASE occurs, spectra should be broad at short stripe lengths and should become narrow as the excitation length increases. ${ }^{22,23}$

Figure 3 shows the spectra recorded for various pump powers for stripe lengths 2,4 , and $6 \mathrm{~mm}$. As the pump stripe length increases, the threshold for ASE decreases. Also, for a given pump energy, the FWHM decreases with increase in stripe length (see Table 1), with a slight shift in the peak wavelength.
Table 1. FWHM of Spectral Emission for Various Pump Energies and Beam Stripe Lengths

\begin{tabular}{cccc}
\hline & \multicolumn{3}{c}{ FWHM (nm) } \\
\cline { 2 - 4 } $\begin{array}{c}\text { Pump Energy } \\
\text { (mJ/pulse) }\end{array}$ & $l=2 \mathrm{~mm}$ & $l=4 \mathrm{~mm}$ & $l=6 \mathrm{~mm}$ \\
\hline 0.031 & $\sim 50$ & 6.6 & 3.4 \\
3.1 & 8.1 & 5.6 & 2.5 \\
9.1 & 5.1 & 4.6 & 1.0 \\
\hline
\end{tabular}

\section{B. Gain Measurement}

According to the method introduced by Shaklet et al., ${ }^{24}$ the relation between the output light varia. tion with excitation length and the gain is given by

$$
I_{0}(l)=\frac{I_{s} A}{g}[\exp (g l)-1],
$$

where $I_{s}$ is the spontaneous emission rate per unit volume; $A$ is a constant related to the spontaneous emission cross section; $g$ is the net gain given by $g^{\prime}$ $-\alpha$, where $g^{\prime}$ is the gain due to stimulated emission; $\alpha$ is the optical loss; and $l$ is the length of the pump stripe.

Shank et al. ${ }^{25}$ measured the single pass gain of dpe laser by comparing the intensities of ASE in single and double cell lengths. By writing an equation sint ilar to that of Eq. (1) for a pump stripe of length $1 / 2$ we get

$$
I_{0}(l / 2)=\frac{I_{s} A}{g}[\exp (g l / 2)-1]
$$

From Eqs. (1) and (2), the net gain can be obtained

$$
g=\frac{2}{l} \ln \left[\frac{I_{0}(l)}{I_{0}(l / 2)}-1\right]
$$

Equation (3) applies only for pump powers up to the onset of saturation The gain at $570 \mathrm{~nm}$ for a pump energy of $0.31 \mathrm{~mJ} /$ pulse calculated as per $\mathrm{Eq}$. (3) wat obtained to be $5.36 \mathrm{~cm}^{-1}$.

\section{Amplified Spontaneous Emission Propagation Through the Waveguide and Wavelength Tuning}

It is well known that by varying the dye concentro tion, wavelength tuning can be attained in dye 1 sers. ${ }^{16}$ For RhG6, there is a spectral region of overtsp between the absorption and emission bands. As $:$ result, the short wavelength emission from the dgr molecules gets absorbed by itself and is reemitted $a^{\prime \prime}$ a longer wavelength. Due to this self-absorption and reemission process, the peak emission wavelengti shows a redshift with an increase in concentration." This property is utilized in the concentration deper. dent tuning of the emitted radiation. Increasing the path length through the dye-doped sample is somewhat similar to an increment in dye concentration. 1 


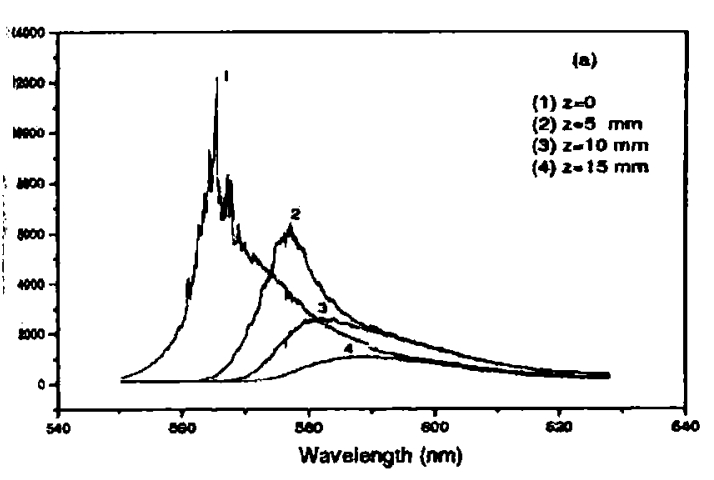

(a)

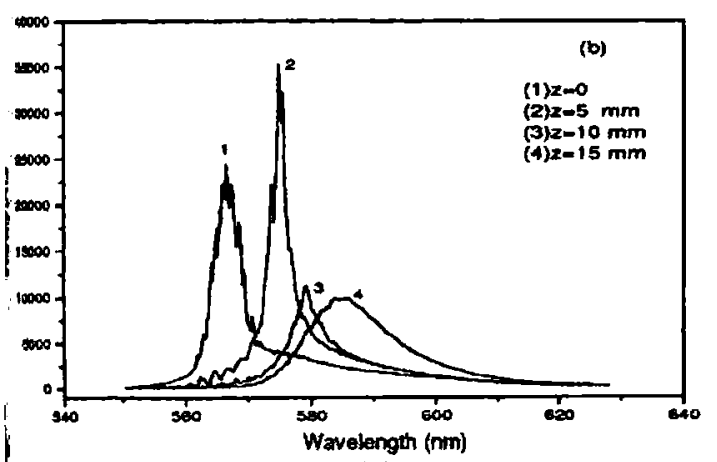

(b)

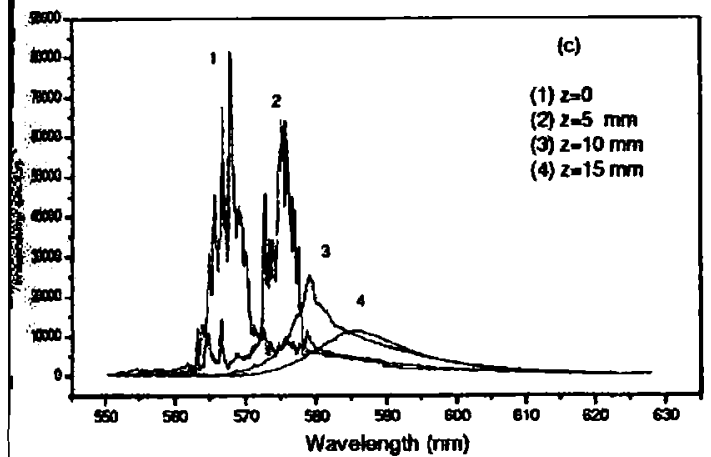

(c)

4. Evolution of ASE as it is guided through various lengths migh the waveguide for stripe lengths: (a) $l=2 \mathrm{~mm}$, (b) $l=4$ if and (c) $l=6 \mathrm{~mm}$ for pump energy $9.1 \mathrm{~mJ} /$ pulse.

inlar redshift is observed when the path length bough the waveguide or fiber is increased. 19-21 $^{-21}$
When irradiated with a narrow stripe, the ASE will be maximum at the stripe ends, along the transverse direction. We changed the pasition of the excitation stripe by translating the waveguide horizontally across the exciting beam. Now the ASE emitted at the end of the stripe is made to traverse through the sample. By shifting the position of the exciting stripe, we are varying the propagation length of the emission through the waveguide. Emitted light is collected exactly in a similar manner as described above. Figure 4 shows the spectra obtained for various $z$ values at a pump energy of $9.1 \mathrm{~mJ} /$ pulse for various stripe lengths.

We observe a redshift for the emission peak with increase in length of propagation. Even after propagation through the sample, we get a narrow emission spectrum, especially for longer stripe lengths. Table 2 gives the values for FWHM and peak emission wavelengths for various pump stripe positions, for the three different stripe lengths corresponding to a pump energy of $9.1 \mathrm{~mJ} /$ pulse.

We see that for a stripe length of $2 \mathrm{~mm}$, we get narrow emission peaked at 565.4 for propagation distances $z=0$ and at $577.2 \mathrm{~nm}$ for $z=5 \mathrm{~mm}$. The spectral width increases from $\sim 5$ to $11 \mathrm{~nm}$ when $z$ changes from 0 to $5 \mathrm{~mm}$. Thus the ASE peak wavelength can be continuously tuned over $12 \mathrm{~nm}$ by changing the $z$ value from 0 to $5 \mathrm{~mm}$ (see Table 1). For further propagation lengths, the emission becomes very broad and ASE character of the emission is lost. With longer pump stripe lengths, the tunability range gets enhanced to around $18 \mathrm{~nm}$. The tuning range can be effectively further enhanced by collecting emission from both ends of the waveguide simultaneously. Details of this will appear elsewhere. ASE can be tuned from 566.6 to 585.4 and 568.1 to 586.1 for stripe lengths 4 and $6 \mathrm{~mm}$, respectively. It should also be noted that during the tuning the spectral width generally increases up to $16 \mathrm{~nm}$. An exception is observed in the case of stripe length $4 \mathrm{~mm}$ at $z$ $=5 \mathrm{~mm}$, for which the intensity corresponding to $z$ $=5 \mathrm{~mm}$ is greater than that corresponding to $z=0$. A similar tendency is observed in the case of stripe length $z=6 \mathrm{~mm}$ for which the peak intensities corresponding to $z=0$ and $z=5 \mathrm{~mm}$ are comparable. This is a clear indication of the existence of gain in the medium. Detailed studies in this direction are in progress.

One of the important points to be noted is that the

Table 2. FWHM and Poak Wovelength for Different $z$ Values at Pump Energy $9.1 \mathrm{~mJ} /$ pulse

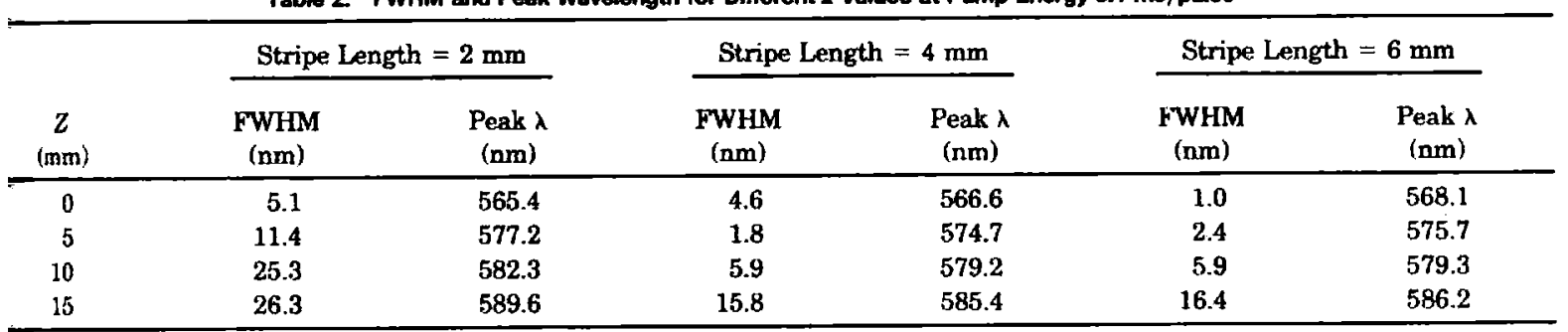



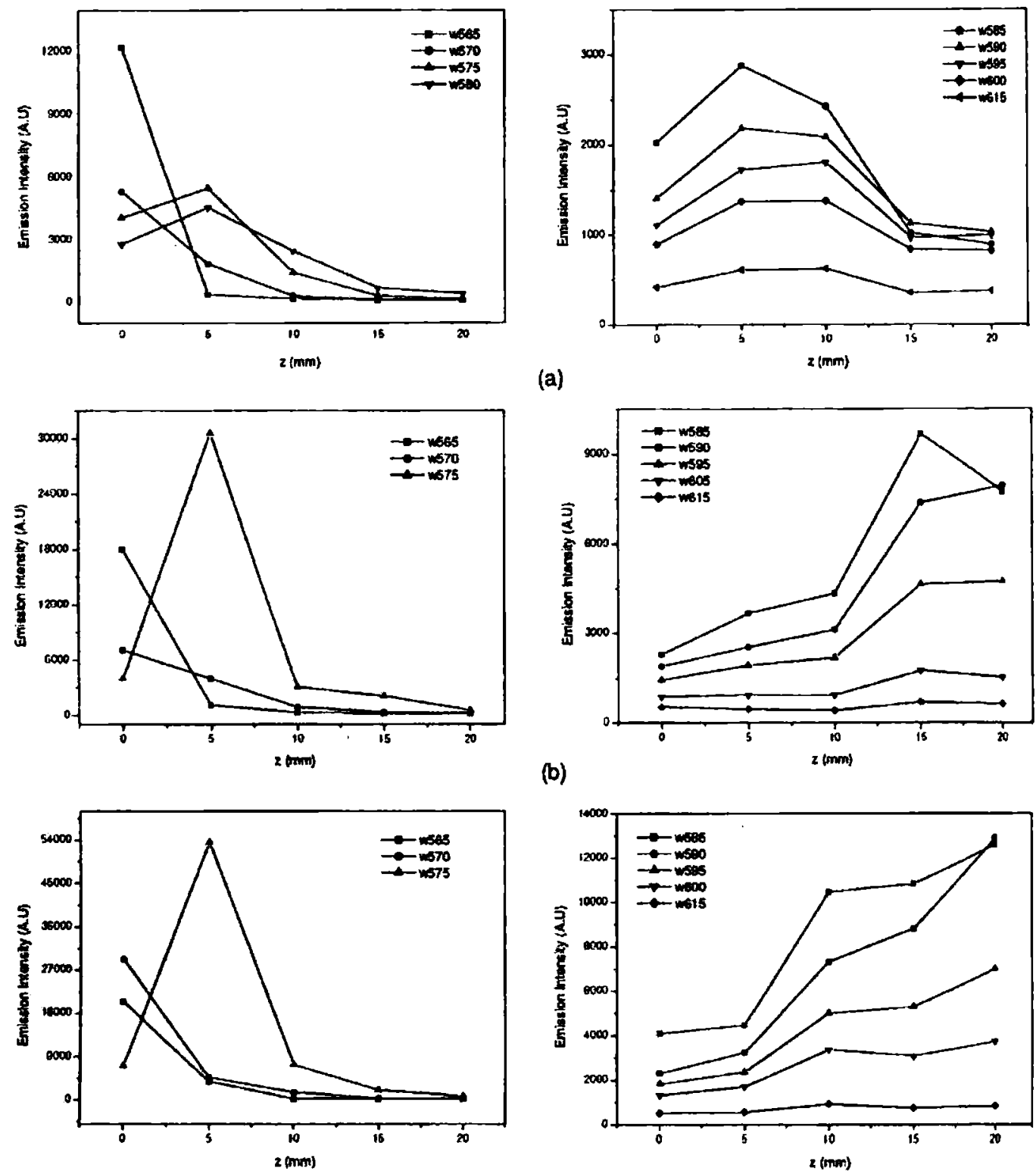

(c)

Fig. 5. Emitted power at various wavelengths versus propagation length through the waveguide for stripe lengths: (a) $l=2 \mathrm{~mm}$ ll $l=4 \mathrm{~mm}$, and (c) $l=6 \mathrm{~mm}$ for pump energy $9.1 \mathrm{~mJ} /$ pulse.

intensity at longer wavelengths of the ASE gets amplified on traversing through the waveguide. Output intensity at shorter wavelengths show the usual exponential decrease. Figure 5 shows the variation of output power at different wavelengths with propagation length through the waveguide.

As can be clearly seen from Fig. 5, as the propagation distance $z$ increases, the emission intensity shows enhancement in the long wavelength region. This gain in the long wavelength region can be attributed to the gain achieved as the radiation is propagated through the amplifying medium as well as due to the reemission from the interacting dye molecules at longer wavelengths. Such emission enhancemeat becomes prominent as the stripe length of the pump beam is increased. Increase in the stripe length hances the interaction length between the pump ro diation and the dye molecules. This will result in the gain in the spectral emission in addition to the gain due to reemission in the long wavelength region. Propagation characteristics of emitted radiation through the waveguide cannot be described by the usual Lambert-Beer law with single attenuation or gain coefficient. Due to complex interactions between the radiation and dye molecules in the gain media one has to describe a space dependent attenuation 
pigain coefficient. This will be the subject matter of future publication.

\section{Conclusions}

have studied the propagation characteristics of amated spontaneous emission through a transversely mped dye-doped polymer freestanding film waveiide. With the increase in propagation length through maveguide, the emission peak shows a redshift due to fabsorption and reemission by the dye molecules. As radiation is propagated through the amplifying mem, we get spectrally narrow output at longer wavegth, due to the gain achieved at these wavelengths. us we could tune the ASE wavelength. The maximum mability obtained by this method was $\sim 18 \mathrm{~nm}$.

The authors acknowledge the financial support m NUFFIC, Netherlands under the MHO assisnoe to the International School of Photonies. The thors thank A. Denny and Raghu Natarajan, MEET, Thrissur, for providing the dye-doped samw. K. Geetha is grateful to the Council of Scientific ii Industrial Research, New Delhi, for the research llowship. M. Rajesh, V.P. N. Nampoori, and C. P. G. Hllabhan acknowledge University Grants Commisin, New Delhi for financial assistance.

\section{Wrences}

IP. P. Sorokin and J. R. Lankard, "Stimulated emission obraved from an organic dye chloroaluminium phtalocyanine," IBM J. Res Develop. 10, 162-163 (1966).

1B. H. Soffer and B. B. Mc Farland, "Continuously tunable narowband organic dye lasers," Appl. Phys. Lett. 10, 266-267 (1967).

10. G. Peterson and B. B. Snavely, "Stimulated emission from ashlamp-excited organic dyes in polymethyl methacrylate," Appl. Phys. Lett. 12, 238-240 (1968).

I. Costela, I. Garcia-Moreno, J. M. Figuera, F. Amat-Gueria, and R. Sastre, "Polymeric matrices for lasing dyes: recent demlopments," Laser Chem. 18, 63-84 (1998).

1. Costela, I. Garcia-Moreno, C. Gomez, O. Garcia, and R Sastre, "Laser performance of pyrromethene 567 dye in solid polymeric matrices with different cross-linking degrees," J. Appl. Phys. 90, 3159-3166 (2001)

ID. Lo, J. E. Parris, and J. L. Lawless, "Multi-megawatt superratiant emissions from coumarin-doped sol-gel derived silica," Appl. Phys. B 55, 365-367 (1992).

Y. Sorek, R. Reisfeld, I. Finkelstein, and S. Ruschin, "Light amplification in a dye-doped glass planar waveguide," Appl. Phys. Lett. 66, 1169-1171 (1995).

iA. Maslyukov, S. Sokolov, M. Kaivola, K Nyholm, and S Popov, "Solid-state dye laser with modified poly(methyl methacrylate)-doped active elements," Appl. Opt. 34, 15161518 (1995)

II C. Yee, T. Y. Tou, and S. W. Ng, "Hot-press molded poly- (methyl methacrylate) matrix for solid-state dye lasers," Appl Opt. 37, 6381-6385 (1998).

10. A. V. Deshpande and E. B. Namdas, "Lasing action of Rhodamine B in poly acrylic acid films," Appl. Phys. B 64, 419-421 (1997).

11. J. E. Roman and K. A. Winick, "Neodymium-doped glass channel waveguide laser containing an integrated distributed Bragg reflector," Appl. Phys. Lett. 61, 2744-2746 (1992).

12. F. J. Duarte, "Multiple-prism grating solid-state dye laser oscillator: optimized architecture," Appl. Opt. 38, 6347-6349 (1999).

13. Xiao-lei Zhu, D. Lo, "Sol-gel glass distributed feedback waveguide laser," Appl. Phys. Lett. 8016), $917-919$ (2002).

14. D. Lo, L. Shi, J. Wang, and G. X. Zhang, "Zirconia and zirconiaorganically modified silicate distributed feedback waveguide lasers tumable in the visible," Appl. Phys. Lett. 81, 2707-2709 (2002).

15. X.1. Zhu and D. Lo, "Temperature tuning of output wave length for solid-state dye lasers," J. Opt. A: Pure Appl. Opt. 3, 225-228 (2001).

16. F. P. Schafer, "Principleg of dye laser operation," in F. P. Shäafer, ed., Dye Lasers, Topics in Applied Physics Volume 1 (Springer-Verlag, Berlin, 1990), Chap. 1.

17. A. K. Sheridan, G. A. Tumbull, A. N. Safonov, and I. D. W. Samuel, "Tuneability of amplified spontaneous emission through control of waveguide mode structure in conjugated polymer films, " Phys. Rev. B 62, R11929-R11932 (2000).

18. Xi. Peng, Li. Liu, Ji. Wu, Yi. Li, Zh. Hou, Le. Xu, We. Wang, Fu $\mathrm{Li}$, and $\mathrm{Mi}$. Ye, "Wide-range amplified spontaneous emission wavelength tuning in a solid-state dye waveguide," Opt. Lett. $25,314-316(2000)$.

19. R. J. Kruhlak and M. G. Kuzyk, "Side illumination fluorescence (SIF) spectroscopy studies of aggregation in ISQ dyedoped polymer optical fibers," in Linear Optical Properties of Waveguides and Fibers, S. Ducharme, D. Dunlap, and R. Norwoods, eds., Proc. SPIE 3799, 312-319 (1999).

20. E. De La Rosa-Cruz, C. W. Dirk, O. Rodriguez, and V. M. Castano, "Characterization of fluorescence induced by side illumination of Rhodamine B-doped plastic optical fibers," Fiber Integr. Opt. 20, 457-464 (2001).

21. K. Geetha, M. Rajesh, C. P. G. Vallabhan, V. P. N. Nampoori, and $P$. Radhakrishnan, "Loss characterization in rhodamine 6G-doped polymer film waveguide by side illumination fluorescence," J. Opt. A: Pure Appl. Opt. 6, 379-383 (2004).

22. M. D. McGehee, R. Gupta, S. Veenstra, E. K. Miller, M. A. Diaz-Garcia, and A. J. Heeger, “Amplified spontaneous emis sion from photopumped films of a conjugated polymer," Phys. Rev. B 58, 7035-7039 (1998).

23. M. A. Diaz-Garcia, S. Fernandez De Avila, and M. G. Kuzyk, "Dye-doped polymers for blue organic diode lasers," Appl. Phys. Lett. 80, 4486-4488 (2002).

24. K. L. Shaklee and R. F. Leheny, "Direct determination of optical gain in semiconductor crystals," Appl. Phys. Lett. 18, 475-477 (1971).

25. C. V. Shank, A. Dienes, and W. T. Silfvast, "Single pass gain of exciplex 4-MU and rhodamine 6G dye laser amplifiers," Appl. Phys. Lett. 17, 307-309 (1970). 